PROCEEDINGS OF THE

AMERICAN MATHEMATICAL SOCIETY

Volume 129, Number 5, Pages 1419-1425

S 0002-9939(00)05717-8

Article electronically published on October 24, 2000

\title{
LOCAL COMPLETENESS AND DUAL LOCAL QUASI-COMPLETENESS
}

\author{
JINGHUI QIU
}

(Communicated by Dale E. Alspach)

\begin{abstract}
It is proved that $l^{q}$-completeness $(1<q<\infty)$ is equivalent to $l^{1}$ completeness (defined by Saxon and Sánchez Ruiz), and becomes a new characteristic condition for local completeness. The relationship between dual local completeness, dual local quasi-completeness and the Banach-Mackey property is investigated. For a quasi-Mackey space, dual local quasi-completeness, $c_{0}$ quasi-barrelledness, Ruess' property (quasi-L) and $C$-quasi-barrelledness are equivalent to each other.
\end{abstract}

\section{LOCAL COMPLETENESS}

In this paper, every space will be assumed a Hausdorff locally convex space over the scalar field of real or complex numbers. Let $(E, t)$ be a space; then $(E, t)^{\prime}$, or briefly $E^{\prime}$, denotes the topological dual of $(E, t)$ and $E^{\#}$ denotes the algebraic dual of $E$. Let $E^{\prime \prime}$ denote $\left(E^{\prime}, \beta\left(E^{\prime}, E\right)\right)^{\prime}$. Recall that a space $E$ is locally complete if and only if every bounded closed absolutely convex subset of $E$ is a Banach disk [4. Proposition 5.1.6]. It is easy to see that local completeness is duality invariant. That is to say, if two spaces $(E, t)$ and $(E, s)$ have the same topological dual and one is locally complete, then so is the other. P. Dierolf ([2] or [4, Theorem 5.1.11]) proved that the following conditions on a space $(E, t)$ are equivalent:

(I) $(E, t)$ is locally complete.

(II) The closed absolutely convex hull of every locally null sequence in $(E, t)$ is compact.

(III) The closed absolutely convex hull of every null sequence in $\left(E, \sigma\left(E, E^{\prime}\right)\right)$ is compact in $\left(E, \sigma\left(E, E^{\prime}\right)\right)$.

(IV) The closed absolutely convex hull of every null sequence in $(E, t)$ is compact.

From [4] the proof of Proposition 3.2.12], we also see that $(E, t)$ is locally complete if and only if for each null sequence $\left(x_{n}\right)$ in $(E, t)$ and each scalar sequence $\left(\lambda_{n}\right) \in l^{1}$, the series $\sum_{n=1}^{\infty} \lambda_{n} x_{n}$ converges in $(E, t)$. Recently Saxon and Sánchez Ruiz [6] proved that a space $E$ is locally complete if and only if it is $l^{1}$-complete. Reminiscently of De Wilde [1, Proposition III.1.4 and V.3.2], they defined a space $E$ to be $l^{1}$-complete if, for each bounded sequence $\left(x_{n}\right) \subset E$ and each $\left(\lambda_{n}\right) \in l^{1}$, the series $\sum_{n=1}^{\infty} \lambda_{n} x_{n}$ converges in $E$. We shall extend the concept to $l^{q}$-completeness

Received by the editors September 28, 1998 and, in revised form, August 17, 1999

2000 Mathematics Subject Classification. Primary 46A08.

Key words and phrases. Locally complete, dual locally quasi-complete, Banach-Mackey property, quasi-Mackey spaces. 
$(1 \leq q \leq \infty)$. If $1 \leq p \leq \infty$, let $l^{p}(E)$ denote all sequences $\left(x_{n}\right)$ in $E$ such that $\left(\rho\left(x_{n}\right)\right) \in l^{p}$ for each continuous seminorm $\rho$ on $E$ (see [4. Definition 4.8.1 and 4.8.2]). Thus, for example, $l^{\infty}(E)$ denotes all bounded sequences in $E$.

Definition 1. A space $E$ is said to be $l^{q}$-complete $(1 \leq q \leq \infty)$ if, for each $\left(\lambda_{n}\right) \in l^{q}$ and each $\left(x_{n}\right) \in l^{p}(E)$, the series $\sum_{n=1}^{\infty} \lambda_{n} x_{n}$ converges in $E$, where $\frac{1}{p}+\frac{1}{q}=1$.

Remark 1. When $q=1$ we have $l^{1}$-completeness defined just as in 6 . When $q=\infty$ we have $l^{\infty}$-completeness of $E$, clearly equivalent to the following condition: for every $\left(x_{n}\right) \in l^{1}(E)$, the series $\sum_{n=1}^{\infty} x_{n}$ converges in $E$; i.e., every absolutely Cauchy series converges in $E$. If every unconditionally Cauchy series converges, $E$ is said to be $\sum$-complete $\left.4,5.3\right]$. Here, $\sum_{n=1}^{\infty} x_{n}$ unconditionally Cauchy means that each $\sum_{n \in \sigma} x_{n}$ is arbitrarily close to the origin whenever $\sigma$ is a finite set of sufficiently large positive integers. Clearly, $\sum$-complete $\Rightarrow l^{\infty}$-complete. The converse fails, as shown by the following Example 1.

Example 1. For any $x=\left(\xi_{n}\right) \in c_{0}$, define $\|x\|=\sup _{n}\left|\xi_{n}\right|$. Then $\left(c_{0},\|\|\right)^{\prime}=l^{1}$ and $\left(l^{1}, \beta\left(l^{1}, c_{0}\right)\right)=\left(l^{1},\|\|_{1}\right)$, where for any $y=\left(\eta_{n}\right) \in l^{1},\|y\|_{1}=\sum_{n=1}^{\infty}\left|\eta_{n}\right|$. As is well known, a subset $K$ of $\left(l^{1},\|\|_{1}\right)$ is relatively compact if and only if the following conditions are satisfied:

(I) there is $M>0$ such that $\sum_{n=1}^{\infty}\left|\eta_{n}\right| \leq M$ for any $y=\left(\eta_{n}\right) \in K$;

(II) for any $\epsilon>0$, there is $m_{0} \in N$ such that when $m \geq m_{0}, \sum_{n=m}^{\infty}\left|\eta_{n}\right|<\epsilon$ for every $y=\left(\eta_{n}\right) \in K$.

For any relatively compact subset $K$ of $\left(l^{1},\|\|_{1}\right)$, we define a seminorm $p_{K}$ on $c_{0}$ as follows:

$$
p_{K}(x)=\sup \left\{\left|\sum_{n=1}^{\infty} \xi_{n} \eta_{n}\right|: y=\left(\eta_{n}\right) \in K\right\},
$$

for any $x=\left(\xi_{n}\right) \in c_{0}$. Let $t$ denote the topology on $c_{0}$ generated by all seminorms $p_{K}$ as above. Since $\left(l^{1},\|\|_{1}\right)$ is complete and in $\left(l^{1},\|\|_{1}\right)$ the weakly compact sets and strong compact sets coincide, we may conclude that $\left(c_{0}, t\right)=\left(c_{0}, \tau\left(l^{\infty}, l^{1}\right) \mid c_{0}\right)$. Thus $(E, t):=\left(c_{0}, t\right)$ illustrates what we defined in the next section as a quasiMackey space. Consider a series $\sum_{n=1}^{\infty} x_{n}$ in $E$. If each $x_{n}$ has 1 at the $n$th coordinate and 0's elsewhere, (II) ensures that $p_{K}\left(\sum_{n \in \sigma} x_{n}\right)<\epsilon$ whenever each member $m$ of the finite set $\sigma$ satisfies $m \geq m_{0}$. Thus the series is unconditionally Cauchy but not even weakly convergent, proving $E$ is not $\sum$-complete. On the other hand, given any series in $E$ with $\sum_{n=1}^{\infty}\left\|x_{n}\right\|=\infty$, we may choose a sequence $\left(b_{n}\right)$ of posive scalars tending to 0 so slowly that $\sum_{n=1}^{\infty} b_{n}\left\|x_{n}\right\|=\infty$ still holds. We then choose each $y_{n} \in l^{1}$ with $\left\|y_{n}\right\|=b_{n}$ and $\sum_{i=1}^{\infty} x_{n}(i) \cdot y_{n}(i)=\left\|x_{n}\right\| \cdot\left\|y_{n}\right\|_{1}=b_{n}\left\|x_{n}\right\|$. The null sequence $\left(y_{n}\right)$ is a relatively compact set $K$ in $\left(l^{1},\|\|_{1}\right)$ such that each $p_{K}\left(x_{n}\right) \geq\left\|x_{n}\right\| b_{n}$, which implies that $\sum_{n=1}^{\infty} p_{K}\left(x_{n}\right)=\infty$. This proves that any absolutely Cauchy series in $(E, t)$ is also absolutely Cauchy and then convergent in the Banach space $\left(c_{0},\|\|\right)$, and therefore convergent in the weaker topology $t$. We conclude that the non- $\sum$-complete $(E, t)$ is $l^{\infty}$-complete.

Remark $2 . l^{\infty}$-completeness certainly implies $l^{1}$-completeness, equivalently, local completeness [6], but the converse is false. Indeed, Pérez Carreras and Bonet [4] Example 5.1.12] noted that. By duality invariance, $E:=\left(c_{0}, \sigma\left(c_{0}, l^{1}\right)\right)$ is locally complete but is not $l^{\infty}$-complete since the canonical unit vectors are not summable in $E$. (They put "sequentially complete" in place of " $l$-complete".) 
It is somewhat surprising that $l^{q}$-completeness $(1<q<\infty)$ is equivalent to $l^{1}$-completeness. Thus $l^{q}$-completeness $(1<q<\infty)$ becomes a new characteristic condition for local completeness.

Theorem 1. For any space $E$ the following statements are equivalent:

(I) $E$ is locally complete.

(II) (Saxon and Sánchez Ruiz ) $E$ is $l^{1}$-complete.

(III) $E$ is $l^{q}$-complete $(1<q<\infty)$.

Proof. The equivalence of (I) and (II) is Theorem 2.1 of [6].

(III) $\Rightarrow$ (II): If $\left(\lambda_{n}\right) \in l^{1}$ and $\left(x_{n}\right) \in l^{\infty}(E)$, then for $1<q<\infty$ and $\frac{1}{p}+\frac{1}{q}=1$ we have

$$
\sum_{n=1}^{\infty} \lambda_{n} x_{n}=\sum_{n=1}^{\infty}\left|\lambda_{n}\right|^{\frac{1}{q}}\left|\lambda_{n}\right|^{\frac{1}{p}}\left(\operatorname{sgn} \lambda_{n}\right) x_{n}
$$

converges in $E$ by $(\mathrm{III})$, since $\left(\left|\lambda_{n}\right|^{\frac{1}{q}}\right) \in l^{q}$ and $\left(\left|\lambda_{n}\right|^{\frac{1}{p}}\left(\operatorname{sgn} \lambda_{n}\right) x_{n}\right) \in l^{p}$.

$(\mathrm{II}) \Rightarrow(\mathrm{III})$ : Suppose $\left(\lambda_{n}\right) \in l^{q}$ and $\left(x_{n}\right) \in l^{p}(E)$, with $p$ and $q$ as above. Since $\sum_{n=1}^{\infty}\left|\lambda_{n}\right|^{q}<\infty$, we may inductively find $1=m_{1}<m_{2}<\cdots$ such that, defining $\sigma_{k}=\left\{n \in N: m_{k} \leq n<m_{k+1}\right\}$ for $k=1,2, \cdots$, we have

$$
\sum_{n \in \sigma_{k}}\left|\lambda_{n}\right|^{q} \leq 2^{-k q} \text { for } k=2,3, \cdots
$$

We must use (II) to show that $\sum_{n=1}^{\infty} \lambda_{n} x_{n}$ converges in $E$. The series is absolutely Cauchy by the Hölder inequality, so we need only show that its sequence of partial sums has a convergent subsequence. Define $y_{k}=2^{k} \sum_{n \in \sigma_{k}} \lambda_{n} x_{n}$ for $k \geq 1$. Given a continuous seminorm $\rho$, the sum $\left(\sum_{n=1}^{\infty}\left[\rho\left(x_{n}\right)\right]^{p}\right)^{\frac{1}{p}}:=M$ is finite. Thus for $k \geq 2$ the triangle and Hölder inequalities imply that

$$
\rho\left(y_{k}\right) \leq 2^{k}\left(\sum_{n \in \sigma_{k}}\left|\lambda_{n}\right|^{q}\right)^{\frac{1}{q}}\left(\sum_{n \in \sigma_{k}}\left[\rho\left(x_{n}\right)\right]^{p}\right)^{\frac{1}{p}} \leq 2^{k}\left(2^{-k q}\right)^{\frac{1}{q}} M=M .
$$

Hence $\left(y_{k}\right) \in l^{\infty}(E)$. Now $\left(2^{-k}\right) \in l^{1}$ and (II) implies that $\sum_{k=1}^{\infty} 2^{-k} y_{k}$ converges in $E$, so its sequence of partial sums is the desired convergent subsequence.

We end this section with a brief discussion of the relationship between convex compactness (cc) and various notions of completeness. Recall that a space $E$ is said to have the convex compactness property if the closed absolutely convex hull of every compact subset of $E$ is still compact [10, Definition 9-2-8]. In [4, 5.3], $E$ is said to be p-complete (in [10, Problem 6-5-107], N-complete; see [3. §23, 9(1)]) if every precompact subset of $E$ is relatively compact; i.e. every closed totally bounded set is compact. Surely, quasi-complete $\Rightarrow$ p-complete, Wheeler denied the converse [10, Table 4], p-complete $\Rightarrow c c$ [10, Problem 9-2-111], and $\mathrm{cc} \Rightarrow$ locally complete by (iv) of Dierolf's Theorem. An amplified Pérez Carreras/Bonet scheme (see [4, 5.3]) emerges:

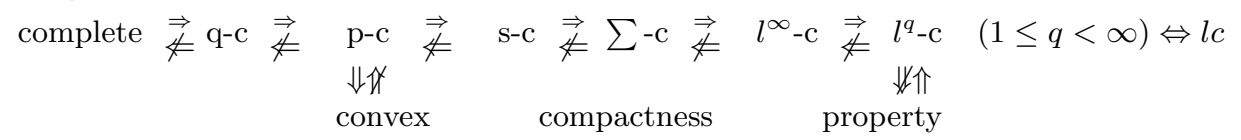

Here, q-c, p-c, s-c, $\sum-\mathrm{c}, l^{\infty}-\mathrm{c}, l^{q}-\mathrm{c}$ and lc respectively denote "quasi-complete", "p-complete", "sequentially complete", " $\sum$-complete", " ${ }^{\infty}$-complete", " $q_{-}$ complete" and "locally complete". The convex compactness property cannot be 
better placed, since s-c $\nRightarrow \mathrm{cc} \nRightarrow l^{\infty}$-c. Indeed, Ostling and Wilansky found an s-c space without the cc property [10. Problem 9-2-301], and $\left(c_{0}, \sigma\left(c_{0}, l^{1}\right)\right)$ has the cc property [10, Theorem 14-2-4] but is not $l^{\infty}$-c (Remark 2). All eight notions of completeness coincide under metrizability [4, Corollary 5.1.9].

\section{Dual local Quasi-COMPleteness}

Recall that a space $E$ is dual locally complete if $\left(E^{\prime}, \sigma\left(E^{\prime}, E\right)\right)$ is locally complete (cf. 6]). Saxon and Sánchez Ruiz [6] investigated dual local completeness and obtained a number of interesting results. Each characterization of local completeness gives one of dual local completeness and vice versa, since $[E$ is locally complete] $\Leftrightarrow$ $\left[\left(E, \sigma\left(E, E^{\prime}\right)\right)\right.$ is locally complete $] \Leftrightarrow\left[\left(E^{\prime}, \sigma\left(E^{\prime}, E\right)\right)\right.$ is dual locally complete $]$. Thus, for example, the Ruess characterization in [6, Theorem 2.3(4)] may be written as follows:

(Ruess) A space $E$ is locally complete if and only if, given any $h \in E^{\prime \#}$ and any absolutely convex absorbing set $A$ in $E^{\prime}$ such that $\left.h\right|_{A}$ is relatively $\sigma\left(E^{\prime}, E\right)$ continuous, it must be so that $h$ is $\sigma\left(E^{\prime}, E\right)$-continuous; i.e., there exists $x_{0} \in E$ such that $h=\hat{x}_{0}$, or $h(f)=f\left(x_{0}\right)$ for every $f \in E^{\prime}$.

Following Tsirulnikov [9], a space $E$ is called dual locally quasi-complete if its strong dual $\left(E^{\prime}, \beta\left(E^{\prime}, E\right)\right)$ is locally complete. Tsirulnikov, Ruess, et al. surely knew that dual locally complete (dlc) $\Rightarrow$ dual locally quasi-complete (dlqc); indeed, this follows from [4. Proposition 5.1.6(iv)], or we may observe that if $\sum_{n=1}^{\infty} \lambda_{n} f_{n}$ converges to $f$ in $\left(E^{\prime}, \sigma\left(E^{\prime}, E\right)\right)$ with $\left(\lambda_{n}\right) \in l^{1}$, and if $\left(f_{n}\right)$ is $\beta\left(E^{\prime}, E\right)$-bounded, then, routinely, $\sum_{n=1}^{\infty} \lambda_{n} f_{n}$ converges to $f$ uniformly on bounded sets of $E$, i.e. in $\left(E^{\prime}, \beta\left(E^{\prime}, E\right)\right)$.

Let $(E, t)$ be a space. An increasing sequence $\sigma=\left\{A_{n}: n \in N\right\}$ of absolutely convex subsets of $E$ is said to be absorbing (bornivorous) if for every $x$ in $E$ (every bounded subset $B$ of $E$ ) there is a positive integer $m$ such that $A_{m}$ absorbs $x$ (absorbs $B$ ); see [4, Definition 8.1.15]. We denote by $t_{\sigma}$ the finest locally convex topology on $E$ that induces the same topology as $t$ on each $A_{n}$. The topology $t_{\sigma}$ is defined by the family of those seminorms whose restrictions to the sets $A_{n}$ are continuous for the topology induced on $A_{n}$ by $t$ (cf. [6]). Absorbing and bornivorous sequences of absolutely convex sets were considered by Valdivia, De Wilde, Houet, Garling, Roelcke, Ruess, et al. (cf. [4, 8.9]). Ruess [5] defined a space $(E, t)$ to have property ([quasi-]L) if $t=t_{\sigma}$ holds for each absorbing [bornivorous] sequence $\sigma$, and to have the weaker property ([quasi- $] L C)$ if each $t_{\sigma}$ is compatible with the dual pair $\left(E, E^{\prime}\right)$. We remark that in the above definitions of Ruess' four properties $([$ quasi- $] L)$ and $([q u a s i-] L C)$, it makes no difference whether the absorbing [bornivorous] sequences are required to be closed or not, by 4, Proposition 8.1.17(i)].

Ruess' characterizations of dual local [quasi-] completeness also make it clear that dlc $\Rightarrow$ dlqc. Please refer to [4, Proposition 8.1.29] and [6, Theorem 2.3].

Theorem 2 (Ruess). For any space $E$ the following statements are equivalent:

(I) $E$ is dual locally [quasi-]complete.

(II) $E$ has property ([quasi-]LC).

(III) If $f \in E^{\#}$ such that $\left.f\right|_{A}$ is continuous, where $A$ is a [bornivorous] barrel in $E$, then $f \in E^{\prime}$. 
A modern statement of the Banach-Mackey Theorem [6] is that every dlc space $E$ has the Banach-Mackey property; i.e., the $\sigma\left(E^{\prime}, E\right)$-bounded sets are $\beta\left(E^{\prime}, E\right)$ bounded, or, equivalently, barrels in $E$ are bornivores. The converse is evidently true when $E$ is dlqc, and this observation transforms the Banach-Mackey theorem into another characterization of dual local completeness that augments, along with dual $l^{q}$-completeness $(1<q<\infty)$, the collection in [6].

Theorem 3 (Banach-Mackey). A space $E$ is dual locally complete if and only if it is dual locally quasi-complete and has the Banach-Mackey property. That is to say, a dual locally quasi-complete space is dual locally complete if and only if it has the Banach-Mackey property.

When $E$ is a Mazur space [10, Definition 8-6-3], then $\left(E^{\prime}, \beta\left(E^{\prime}, E\right)\right)$ is complete [10, Corollary 8-6-6], and is certainly locally complete; i.e., Mazur $\Rightarrow$ dlqc. By Theorem 3, then, a Mazur space is dlc if and only if it has the Banach-Mackey property. This is just [6. Theorem 2.6], viewed now as a corollary to the Banach-Mackey Theorem. Note that $E$ need not be a Mazur space even though $\left(E^{\prime}, \beta\left(E^{\prime}, E\right)\right)$ is complete; e.g., take $E=\left(l^{1}, \tau\left(l^{1}, c_{0}\right)\right)$ [10, Problem 8-6-119]. Hence, Theorem 3 is a useful characterization of dual local completeness which properly extends $[\underline{6}$. Theorem 2.6].

The observations that

(I) $E$ is dlc $\Rightarrow E$ is dlqc $\Leftrightarrow\left(E^{\prime \prime}, \sigma\left(E^{\prime \prime}, E^{\prime}\right)\right)$ is dlc, and

(II) each $\sigma\left(E^{\prime}, E\right)$-bounded set is $\beta\left(E^{\prime}, E^{\prime \prime}\right)$-bounded $\Leftrightarrow$ both $E,\left(E^{\prime \prime}, \sigma\left(E^{\prime \prime}, E^{\prime}\right)\right)$ have the Banach-Mackey property

combine with Theorem 3 as follows.

Corollary 1. In a dlc space $E$, each $\sigma\left(E^{\prime}, E\right)$-bounded set is $\beta\left(E^{\prime}, E^{\prime \prime}\right)$-bounded.

Remark 3. Banach-Mackey $\stackrel{\nRightarrow}{\sharp}$ dlqc: Trivially, any non-barrelled normed space is dlqc but is without the Banach-Mackey property. Conversely, let $(X,\|\|)$ be any barrelled normed space which is not complete [10, Problem 3-1-4], equivalently, not lc, and put $E:=\left(X^{\prime}, \tau\left(X^{\prime}, X\right)\right)$. Then $\left(E^{\prime}, \beta\left(E^{\prime}, E\right)\right)=\left(X, \beta\left(X, X^{\prime}\right)\right)=(X,\|\|)$ is not locally complete; i.e. $E$ is not dlqc. But $E$ does have the Banach-Mackey property, since $\sigma\left(E^{\prime}, E\right)$-bounded $\Leftrightarrow \sigma\left(X, X^{\prime}\right)$-bounded $\Leftrightarrow \beta\left(X, X^{\prime}\right)$-bounded $\Leftrightarrow$ $\beta\left(E^{\prime}, E\right)$-bounded.

Remark 4. Note in the above that $E=E^{\prime \prime}$. Therefore, [each $\sigma\left(E^{\prime}, E\right)$-bounded set is $\beta\left(E^{\prime}, E^{\prime \prime}\right)$-bounded $] \nRightarrow[E$ is dlqc $]$.

Mazon defined a space $E$ to be $C$-[quasi-]barrelled [4, Definition 8.2.6] if $U:=$ $\bigcap_{n=1}^{\infty} U_{n}$ is a neighborhood of 0 whenever $\left(U_{n}\right)$ is a sequence of absolutely convex closed neighborhoods of 0 such that any given singleton [bounded] set is contained in $U_{n}$ for almost all $n$. According to Webb, $(E, t)$ is $c_{0}$-[quasi-]barrelled if each $\sigma\left(E^{\prime}, E\right)$-null [ $\beta\left(E^{\prime}, E\right)$-null] sequence is $t$-equicontinuous. It is known [4, Proposition 8.1.29, Observation 8.2.7 and 8.2.23] that $C$-[quasi-]barrelled implies both $c_{0}$-[quasi-]barrelled and Ruess' property ([quasi-] $L$ ), either of which implies dual locally [quasi-]complete, respectively. Furthermore, each one of the four quasiexclusive conditions implies the corresponding quasi-inclusive condition. It has also been known for some time that, in both the quasi-inclusive and -exclusive cases, the latter three notions coincide under the assumption of the Mackey topology (see [4] Proposition 8.1.29 and Observation 8.2.23(c)]). Recently, Saxon and Sánchez Ruiz 
7] showed that [Mackey and dlc] $\Rightarrow[C$-barrelled], so that, in the quasi-exclusive case, all four notions coincide for Mackey spaces. Their proof applies equally well to the inclusive case.

Theorem 4 (Saxon and Sánchez Ruiz). For a Mackey space $E$ the following are respectively equivalent:

(a) $E$ is dual locally [quasi-]complete.

(b) $E$ is $c_{0}$-[quasi-]barrelled.

(c) E has property $([q u a s i-] L)$.

(d) $E$ is $C$-[quasi-]barrelled.

Thus a space $E$ with dual local quasi-completeness, the weakest of the four "quasi" properties, suddenly enjoys $C$-quasi-barrelledness, the strongest of the four, when it is endowed with its Mackey topology. Let us identify another such compatible topology.

Definition 2. A space $E$ is called a quasi-Mackey space if it has its quasi-Mackey topology; i.e., the topology induced by $\left(E^{\prime \prime}, \tau\left(E^{\prime \prime}, E^{\prime}\right)\right)$.

Köthe [3, §23,4.(6)] noted that the quasi-Mackey topology is compatible with the pairing $\left(E, E^{\prime}\right)$ and may be strictly coarser than the Mackey topology $\tau\left(E, E^{\prime}\right)$, e.g., when $E=c_{0}$ with $E^{\prime}=l^{1}$. This permits a new parallel to Theorem 4's quasi-inclusive case.

Theorem 5. For a quasi-Mackey space $E$ the following statements are equivalent:

(a) $E$ is dual locally quasi-complete.

(b) $E$ is $c_{0}$-quasi-barrelled.

(c) E has property (quasi-L).

(d) $E$ is $C$-quasi-barrelled.

Proof. It suffices to show that (a) $\Rightarrow(\mathrm{d})$ : Suppose $U:=\bigcap_{n=1}^{\infty} U_{n}$ is given as in the definition of $C$-quasibarrelled. For $A \subset E^{\prime}$, let $A^{\circ}$ and $A^{\bullet}$ denote the polar of $A$ in $E$ and $E^{\prime \prime}$, respectively. Since $E$ is quasi-Mackey, for each $U_{n}$ there is an absolutely convex $\sigma\left(E^{\prime}, E^{\prime \prime}\right)$-compact set $C_{n}$ such that $U_{n} \supset C_{n}^{\bullet} \cap E=C_{n}^{\circ}$. As $C_{n}$ is also $\sigma\left(E^{\prime}, E\right)$-compact and hence $\sigma\left(E^{\prime}, E\right)$-closed, we have $C_{n}=C_{n}^{\circ} \supset U_{n}^{\circ}$. Therefore $C_{n}^{\bullet} \subset U_{n}^{\circ \bullet}$, and the latter is a 0-neighborhood in $\left(E^{\prime \prime}, \tau\left(E^{\prime \prime}, E^{\prime}\right)\right)$. Given $z \in E^{\prime \prime}$, there exists a bounded set $B \subset E$ such that $z \in B^{\circ \bullet}$, and by definition, $B \subset U_{n}$ for almost all $n$. Therefore $z \in U_{n}^{\circ \bullet}$ for almost all $n$. Duality invariance and (a) imply that $\left(E^{\prime \prime}, \tau\left(E^{\prime \prime}, E^{\prime}\right)\right)$ is dlc; hence $C$-barrelled, by Theorem 4 . It follows that $W:=\bigcap_{n=1}^{\infty} U_{n}^{\circ \bullet}$ is a 0-neighborhood in $\left(E^{\prime \prime}, \tau\left(E^{\prime \prime}, E^{\prime}\right)\right)$, and thus

$$
W \bigcap E=\bigcap_{n=1}^{\infty}\left(U_{n}^{\circ \bullet} \cap E\right)=\cap_{n=1}^{\infty} U_{n}^{\circ \circ}=U
$$

is a 0 -neighborhood in the quasi-Mackey space $E$.

By definition, a $c_{0}$-quasi-barrelled space remains so under any finer compatible topology. Dierolf's Theorem and Theorem 5 each implies that if $(E, t)$ is dlqc with $t$ finer than the quasi-Mackey topology, then $(E, t)$ is $c_{0}$-quasi-barrelled. This properly extends the quasi-inclusive case of $(\mathrm{a}) \Leftrightarrow(\mathrm{b})$, Theorem 4 .

Corollary 2. A space whose topology lies between its Mackey and quasi-Mackey topologies is $c_{0}$-quasi-barrelled if and only if it is dual locally quasi-complete. 
We cannot similarly extend the quasi-exclusive case of (a) $\Leftrightarrow$ (b), Theorem 4 : If we take $E=c_{0}$ with $E^{\prime}=l^{1}$ and give $E$ its quasi-Mackey topology, as in Example 1, then $E$ is dlc but is not $c_{0}$-barrelled, since the canonical unit vectors are not equicontinuous on $E$. A fortiori, $E$ is not $C$-barrelled. Yet $E$ does have property $(L)$, as noted in [8]. In fact, Theorem 5 provides a general proof: $E$ has property (quasi- $L$ ), and the Banach-Mackey property says that every closed absorbing sequence is bornivorous [6, Theorem 2.4].

Corollary 3. A quasi-Mackey space has property $(L)$ if and only if it is dual locally complete.

\section{Acknowledgement}

The author is very grateful to the referee for many valuable comments and suggestions.

\section{REFERENCES}

[1] M. De Wilde, Closed Graph Theorems and Webbed Spaces, Pitman, 1978. MR 81j:46013

[2] P. Dierolf, Une characterisation des espaces vectoriels complets au sens de Mackey, C. R. Acad. Sci. Paris. 283 (1976), 245-248.

[3] G. Köthe, Topological Vector Spaces I, Springer-Verlag, 1969. MR 40:1750

[4] P. Pérez Carreras and J. Bonet, Barrelled Locally Convex Spaces, Math. Studies 131, North Holland, 1987. MR 88j:46003

[5] W. Ruess, A Grothendieck representation for the completion of cones of continuous seminorms, Math. Ann. 208(1974), 71-90. MR 50:14147

[6] S. A. Saxon and L. M. Sánchez Ruiz, Dual local completeness, Proc. Amer. Math. Soc. 125(1997), 1063-1070. MR 97g:46004

[7] S. A. Saxon and L. M. Sánchez Ruiz, Mackey weak barrelledness, Proc. Amer. Math. Soc. 126(1998), 3279-3282. MR 99a:46006

[8] S. A. Saxon and L. M. Sánchez Ruiz, Reinventing weak barrelledness, preprint.

[9] B. Tsirulnikov, On conservation of barrelledness properties in locally convex spaces, Bull. Soc. Royale Sci. Liège 49(1980), 5-25. MR 82c:46005

[10] A. Wilansky, Modern Methods in Topological Vector Spaces, McGraw-Hill, 1978. MR 81d:46001

Department of Mathematics, Suzhou University, Suzhou 215006, People's Republic OF CHINA 